\title{
A cluster expansion for interacting spin-flip processes
}

\author{
Massimo Campanino ${ }^{1, \star}$ and Michele Gianfelice ${ }^{2, \star \star}$ \\ ${ }^{1}$ Dipartimento di Matematica, Università degli Studi di Bologna, P.zza di Porta San Donato 5, 40126 Bologna, Italy \\ ${ }^{2}$ Dipartimento di Matematica, Università della Calabria, Campus di Arcavacata, Ponte P. Bucci - cubo 30B, 87036 Arcavacata di \\ Rende, Italy
}

\begin{abstract}
We consider a system of spin flip processes, one-for each point of $\mathbb{Z}$, interacting through an Ising type interaction. We construct a cluster expansion and prove that it is convergent when the intensity $h$ of the spin-flip processes is sufficiently high. The system is relevant in the study of the ground state of a quantum Ising process with transverse magnetic field.
\end{abstract}

\section{Introduction}

The ground state of the Quantum Ising model with a transverse magnetic field can be represented as a classical Ising model with one added continuous dimension ([DLP]). In its turn this classical Ising model can be represented via a suitable FK random cluster model ([F] [CKP]). This last representation has been used for example in [GOS] to study the entaglement of the ground state.

Here we consider an Ising model in $1+1$ dimensions, where the second dimension is continuous. We introduce a representation of this model as a Gibbs random field in $\mathbb{Z}^{2}$, in which the spins take values in a space of trajectories. We construct a cluster expansion based on this representation and prove that it satisfies the conditions for convergence (see [KP] when the parameter $h$ corresponding to the transverse magnetic field is sufficiently large.

\section{Definition of the system}

We consider a Gibbs random field on $\mathbb{Z}$ in which the spins take values in a space of processes with values in $\{-1,1\}$.

Let us first define the finite volume distribution. We consider on $\mathbb{R}$ a Poisson point process with intensity $h$. Given an interval $\left[-\frac{\beta}{2}, \frac{\beta}{2}\right]$, the Poisson point process induces a measure on piecewise constant functions on $\left[-\frac{\beta}{2}, \frac{\beta}{2}\right]$ with values in $\{-1,1\}$. The spin configuration $\sigma(t)$ at $t= \pm \frac{\beta}{2}$ is obtained by taking the values equal to -1 or 1 with probability $\frac{1}{2}$ and switching the value of $\sigma(t)$ at each point of the point process configuration. It is not important which extreme of $\left[-\frac{\beta}{2}, \frac{\beta}{2}\right]$ one chooses and which value one assigns to the spin at switwing times..

\footnotetext{
^e-mail: Massimo.Campanino@unibo.it

$\star \star$ e-mail: gianfelice@mat.unical.it

${ }^{0}$ AMS Subject Classification : 60K35, 60F15, 60K15, 82B43.

Keywords and phrases: Spin-flip processes, Gibbs random fields, cluster expansion
}

Let us denote by $X_{I}$ the set of piecewise constant functions with values in $\{-1,1\}$ defined on the interval $I$ and by $\mu_{I}$ the measure just described on $X_{I}$.

Let $\Lambda$ a finite subinterval of $\mathbb{Z}$. We define a boundary condition for $\Lambda \times\left[-\frac{\beta}{2}, \frac{\beta}{2}\right]$ as two configurations $\eta_{1}, \eta_{2}$ in $X_{\left[-\frac{\beta}{2}, \frac{\beta}{2}\right]}$ for the horizontal boundary and two configuration $\xi_{1}, \xi_{2} \in\{-1,1\}$ for the lower and the upper boundary.

The conditional Gibbs measure is a measure on the space $X_{\left[-\frac{\beta}{2}, \frac{\beta}{2}\right]}^{\Lambda}$ whose density w.r.t. $\bigotimes_{i \in \Lambda} \mu_{\left[-\frac{\beta}{2}, \frac{\beta}{2}\right]}^{(i)}$ is given by

$$
\begin{aligned}
& Z_{\eta_{1}, \eta_{2}, \xi_{1}, \xi_{2}}^{-1} \times \\
& \exp \left(-J \sum_{x, y \in \Lambda:|x-y|=1} \int_{-\frac{\beta}{2}}^{\frac{\beta}{2}} d t \sigma_{x}(t) \sigma_{y}(t)\right. \\
& \left.-J \sum_{x \in \Lambda} \sum_{y \in \partial \Lambda:|x-y|=1} \int_{-\frac{\beta}{2}}^{\frac{\beta}{2}} d t \sigma_{x}(t) \eta_{y}(t)\right) \\
& \times \prod_{x \in \Lambda} \delta_{\sigma_{x}\left(-\frac{\beta}{2}\right), \xi_{1}(x)} \delta_{\sigma_{x}\left(\frac{\beta}{2}\right), \xi_{2}(x)}
\end{aligned}
$$

where $Z_{\eta_{1}, \eta_{2}, \xi_{1}, \xi_{2}}$ is the normalizing constant.

We first define a conditional measure on the trajectories $\sigma(t)$ with values in $\{-1,1\}$ for $t$ belonging to some interval $[a, b]$ and boundary conditions $\sigma(a)=\varepsilon_{1}$ and $\sigma(b)=\varepsilon_{2}$ with $\varepsilon_{1}, \varepsilon_{2} \in\{-1,1\}$.

Let $\mu$ be a Poisson point process in the interval $[a, b]$ with intensity $h$. If $\varepsilon_{1}=\varepsilon_{2}, \mu^{\varepsilon_{1}, \varepsilon_{2}}$ is the measure $\mu$ conditioned to the presence of an even number of jumps, while if $\varepsilon_{1} \neq \varepsilon_{2}$ then $\mu^{\varepsilon_{1}, \varepsilon_{2}}$ is the measure $\mu$ conditioned to the presence of an odd number of jumps. Hence the trajectory corresponding to this realization is a piecewise constant function which is equal to $\varepsilon_{1}$ in $a$ and to $\varepsilon_{2}$ in $b$ and has jumps in each point of the point process configuration.

It doesn't matter the value at the jump points since it has no consequence. 


\section{The cluster expansion}

We perform a cluster expansion on the model and verify that, when $h$ is sufficiently large, we can ensure that, for a suitable choice of the parameter, the condition of Kotezky and Preiss are satisfied and the cluster expansion is therefore convergent.

Set $\delta=\frac{\beta}{N}$ for some $N$. We subdivide each "vertical segment" into closed subintervals of lenght $\delta$. We associate a lattice to the subdivision and to each site of the lattice a spin in the space of the piecewise function on the interval $[0, \delta]$ with values in $\{-1,1\}$. The spins on neighbouring sites in the vertical directions must satisfy the compatibility condition that the final value of the trajectory of one spin must agree with the initial value of the trajectory of the other.

Therefore, we consider a spin model on $\mathbb{Z} \times \delta \mathbb{Z}$. If $x=$ $\left(x_{1}, x_{2}\right)$ and $y=\left(x_{1}, x_{2}\right)$ with $x_{2} \in \delta \mathbb{Z}, x_{1}, y_{1} \in \mathbb{Z}$ with $\left|x_{1}-y_{1}\right|=1$, then

$$
\begin{aligned}
& \qquad W\left(\sigma_{x}, \sigma_{y}\right):=J \int_{0}^{\delta} \sigma_{x}(t) \sigma_{y}(t) d t \\
& \text { If } x=\left(x_{1}, x_{2}\right) \text { and } y=\left(x_{1}, y_{2}\right) \text { with } \\
& y_{2}=x_{2}+\delta \text {, then }
\end{aligned}
$$

$$
W\left(\sigma_{x}, \sigma_{y}\right):=\delta_{\sigma_{x}(t), \sigma_{y}(t)} .
$$

The finite volume distribution on a volume $\Lambda$ with boundary conditions $\eta$ on $\partial \Lambda$ has density w.r.t. the reference measure $\bigotimes_{x \in \Lambda} \mu\left(d \sigma_{x}\right)$ given by

$$
Z_{\eta}^{-1} \exp \left(-\sum_{x, y \in \Lambda:|x-y|=1} W\left(\sigma_{x}, \sigma_{y}\right)-\sum_{x \in \Lambda} \sum_{y \in \partial \Lambda} W\left(\sigma_{x}, \eta_{y}\right)\right)
$$

with $Z_{\eta}$ a normalizing constant.

We perform a first expansion: if $x=\left(x_{1}, x_{2}\right)$ and $y=$ $\left(y_{1}, y_{2}\right)$ with $\left|x_{2}-y_{2}\right|=1$, we write

$$
e^{-W\left(\sigma_{x}, \sigma_{y}\right)}=1+\left[e^{-W\left(\sigma_{x}, \sigma_{y}\right)}-1\right]
$$

and perform the expansion

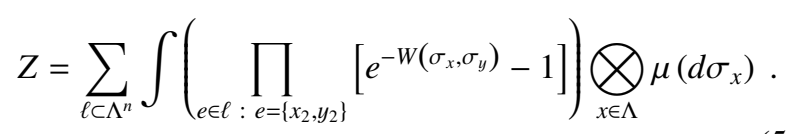

Given $\ell \subset \Lambda^{n}$ on each vertical segment we look at those sites $A$ that belong to some $e \in \ell$. If two sites in $A$ are not consecutive we integrate over over the intermediate sites. This integral can be explicitely performed: if the sites are $x=\left(x_{1}, x_{2}\right)$ and $y=\left(y_{1}, y_{2}\right)$ with $y_{1}>x_{1}$, the integral is

$$
\left\{\begin{array}{ll}
\frac{1+e^{-2 h\left(y_{1}-x_{1}\right)}}{2} & \text { if } \sigma_{x}(\delta)=\sigma_{y}(0) \\
\frac{1-e^{-2 h\left(y_{1}-x_{1}\right)}}{2} & \text { if } \sigma_{x}(\delta) \neq \sigma_{y}(0)
\end{array} .\right.
$$

$R$

We take $\delta=\frac{1}{\sqrt{h}}$. Therefore, the activity of a polymer

can be estimated by

$$
\left(e^{\frac{1}{\sqrt{h}}}-1\right)^{\#\{\text { horizontal bonds\} }} \frac{e^{-2 \sqrt{h} \#\{\text { vertical bonds }\}}}{2} .
$$

If we denote by

$V(R):=\#\{$ horizontal bonds of $R\}+\#\{$ vertical bonds of $R\}$

We have proved the following theorem.

Theorem. The activity of a polymer $\zeta(R)$ can be bounded by

$$
\zeta(R) \leq c(h)^{V(R)}
$$

where

$$
c(h):=\max \left\{e^{\frac{1}{\sqrt{h}}}-1, e^{-2 \sqrt{h}}\right\} .
$$

Remark. The bounds in (9) and (10) imply that cluster expansion (see [KP]) is convergent. In particular they imply that two point correlation functions decay exponentially with the distance when $h$ is large with decay constant going to $\infty$ as $h \rightarrow \infty$.

\section{Conclusions}

The result of this paper can be extended without problems to $\mathbb{Z}^{d}$ and should be appliable to study the ground state of the quantum Ising model with a transverse magnetic flield and in particular the entanglement.

\section{References}

[KP] R. Kotecky, D. Preiss Cluster expansion for abstract polymer models. Comm. Math. Phys. 103, 491-498 (1986)

[CKP] M. Campanino, A. Klein, F. Perez Smoothness of the density of states in the Anderson model at high disorder. Comm. Math. Phys. 114, 439-461 (1988)

[DLP] W. Driessler, L. Landau, J.F. Perez Estimates of critical lengths and critical temperatures for classical and quantum lattice systems J. Statist. Phys. 20123-162 (1979)

[F] C. M. Fortuin et al. On the Random cluster model. 1. Introduction and relation to other models Physica 57 536-564 (1972)

[GOS] G. R. Grimmett, T. J. Osborne, P. F. Scudo Entanglement in the quantum Ising model. J. Statist. Phys. 131 305-339 (2008). 